\title{
Intervenção multiprofissional melhora a aptidão física relacionada à saúde de adolescentes com maior efeito sobre as meninas em comparação aos meninos
}

CDD. 20.ed. 613.398 613.7

http://dx.doi.org/10.1590/1807-55092016000401051
Josiane Aparecida Alves BIANCHINI* Danilo Fernandes DA SILVA* Carlos Andres LOPERA* Vanessa Drieli Seron ANTONINI* Nelson NARDO JUNIOR*
*Centro de Ciências da Saúde, Universidade Estadual de Maringá, Maringá, PR, Brasil.

\section{Resumo}

Este estudo teve como objetivo verificar as diferenças entre as respostas de meninos e meninas a um programa multiprofissional de tratamento da obesidade sobre a aptidão física relacionada à saúde. Fizeram parte do estudo 163 adolescentes com excesso de massa corporal e idade entre 10 e 18 anos. Foram avaliados parâmetros antropométricos, composição corporal, flexibilidade, força e resistência abdominal e aptidão cardiorrespiratória antes e após 16 semanas de intervenção multiprofissional. A intervenção multiprofissional contou com a participação de profissionais de educação física, nutrição, psicologia e pediatria e teve como foco principal o incentivo a mudanças nos hábitos alimentares e de atividade física que pudessem promover alterações positivas nos parâmetros analisados. A intervenção teve frequência semanal de três sessões, sendo uma hora de intervenções teóricas com os profissionais de educação física, nutrição e psicologia e a outra hora destinada à prática regular de exercícios físicos, com predominância de exercícios físicos resistidos, aeróbios e prática de basquetebol. Após a intervenção tanto as meninas quanto os meninos apresentaram melhoras significativas nas variáveis IMC, circunferência de cintura e quadril, massa gorda relativa e absoluta, massa magra, flexibilidade, força/resistência abdominal e aptidão cardiorrespiratória, porém com resultados mais expressivos para as meninas sobre a circunferência de cintura, gordura corporal $(\mathrm{kg}), \mathrm{VO}_{2 \max }$ e força/resistência abdominal.

Palavras-chave: Adolescente; Obesidade; Aptidão física; Estudos de intervenção.

\section{Introdução}

Nas últimas décadas, a obesidade tem se apresentado como uma epidemia global na população jovem, sendo considerada uma importante preocupação em saúde pública devido, principalmente, às comorbidades associadas. Esta doença geralmente está associada à redução dos níveis de aptidão física relacionada à saúde (AFRS), dentre eles da aptidão cardiorrespiratória, que por sua vez pode contribuir para o aumento do risco de doenças cardiometabólicas ${ }^{1-3}$.

Desse modo, estratégias de tratamento são recomendadas assim que identificado o excesso de gordura corporal, buscando aumentar os níveis de AFRS das crianças e adolescentes, a fim de evitar complicaçóes decorrentes do excesso de gordura corporal ${ }^{4-7}$. O aumento dos níveis de aptidão cardiorrespiratória proporciona melhora no estado metabólico de jovens obesos $^{8}$, exercendo um papel cardioprotetor ${ }^{3-4}$.

Intervençōes para crianças e adolescentes com excesso de gordura corporal, focadas em mudança do estilo de vida têm apresentado resultados significativos na $\mathrm{AFRS}^{9-10}$, principalmente em relação à melhora na aptidão cardiorrespiratória ${ }^{11-14}$. Apesar dos resultados positivos observados sobre a AFRS', a partir do que se foi levantado na literatura, não há estudos que tenham investigado se existem diferenças nas respostas de meninos e meninas durante intervençóes 
multidisciplinares envolvendo mudanças no estilo de vida em parâmetros da AFRS. Durante a puberdade espera-se para as meninas um aumento da quantidade de gordura corporal, enquanto para os meninos observase um aumento da massa muscular, contribuindo assim para a melhora de alguns parâmetros da AFRS para o sexo masculino ${ }^{15-16}$. Além das questôes fisiológicas e metabólicas, meninos e meninas costumam apresentar diferenças comportamentais que poderiam interferir nos resultados de um programa de intervenção para o tratamento da obesidade. Assim, ainda não está evidente se meninos e meninas respondem de forma diferente ao tratamento multiprofissional da obesidade. Conhecer as diferenças entre meninos

\section{Método}

\section{Sujeitos}

Fizeram parte do estudo 163 adolescentes com excesso de massa corporal classificados de acordo com os pontos de corte propostos por Cole e LobSTEIN ${ }^{17}$ e idade entre 10 e 18 anos, que foram convidados por meio de divulgação na mídia para participarem do PMTO nos anos de 2009 a 2013.

Foram adotados os seguintes critérios de inclusão para formação do grupo intervenção (GI): concordância do adolescente e seu responsável em participar do PMTO e disponibilidade para participar integralmente das intervenções. Os critérios de exclusão foram: problemas genéticos, endócrinos ou metabólicos que requeriam o uso de quaisquer medicamentos que pudessem interferir no controle do excesso de gordura corporal (exemplo: uso de glicocorticóides ou psicotrópicos) e que foram previamente diagnosticados e informados ao pediatra; consumo em longo prazo de álcool; taxa de adesão de, no mínimo, 70\% das intervenções multiprofissionais ${ }^{18-19}$.

Os adolescentes que não cumpriram especificamente o critério de inclusão relacionado à disponibilidade de participação das intervenções e que não apresentaram nenhum dos critérios de exclusão foram convidados a fazerem parte do grupo controle (GC) do estudo, que foi avaliado antes e após o mesmo período da intervenção, porém, não receberam qualquer orientação da equipe do PMTO ou participaram de programas visando à redução da gordura corporal e só foram contatados para o agendamento das avaliaçôes pós 16 semanas. e meninas quanto aos efeitos de um programa multiprofissional pode auxiliar a compreender se as diferenças fisiológicas e comportamentais entre os dois sexos podem influenciar no resultado desses programas, podendo assim, contribuir para o sucesso do tratamento, trazendo elementos relevantes para se levar em consideração durante o processo interventivo, podendo inclusive levantar a discussão de haver ou não a necessidade de trabalhar com os sexos de maneira diferenciada.

Desse modo, o objetivo do presente estudo foi verificar as diferenças entre as respostas de meninos e meninas a um programa multiprofissional de tratamento da obesidade (PMTO) sobre a AFRS.

Dos 163 adolescentes que completaram o protocolo, 97 (52 meninas - 53,6\%) adolescentes foram alocados para o GI e 66 (30 meninas - 45,5\%) para o GC.

O estudo foi aprovado pelo Comitê de Ética local (protocolo 463/2009) e está de acordo com a declaração de Helsinki.

\section{Protocolo de intervenção multiprofissional}

A intervenção multiprofissional teve duração de 16 semanas, foi realizada duas vezes no ano e contou com uma equipe multiprofissional composta por profissionais de educação física, nutricionistas, uma psicóloga e um médico pediatra. O principal objetivo da equipe foi contribuir com mudanças positivas em variáveis da composição corporal, em médio prazo, a partir do incentivo e prática de atividade física e orientação de hábitos saudáveis relacionados à alimentação, com base na terapia cognitivo comportamental $^{20}$. Os adolescentes frequentaram o PMTO três vezes na semana, segundas, quartas e sextas das 16:00 às 18:00 horas. Na primeira hora (16:00 às 17:00 horas) das segundas-feiras foram realizadas as intervenções teóricas da educação física. Neste mesmo horário nas quartas-feiras os adolescentes participaram das intervenções da nutrição e nas sextas-feiras das intervençôes da psicologia. A segunda hora (17:00 às 18:00 horas) dos três dias da semana foi destinada à intervenção prática da educação física. 


\section{Intervenção psicológica}

Foi conduzida por uma psicóloga especialista em terapia cognitivo comportamental e acadêmicas do curso de psicologia, por meio de uma reunião semanal em grupo, com duraçáo média de uma hora, com a finalidade de abordar os seguintes tópicos: a) estabelecimento de metas; b) auto-observaçáo dos próprios comportamentos e consequências (imediatas, a médio prazo e a longo prazo); c) discriminação de sensaçôes e análise de sentimentos; d) imagem corporal; e) ampliação do autoconhecimento e análise dos eventos internos (pensamentos) e suas consequências; $f$ ) aspectos relacionados à automotivação e autocontrole; g) relacionamentos interpessoais (habilidades sociais).

\section{Intervenção nutricional}

Conduzida por uma nutricionista experiente, por meio de reuniōes semanais em grupo com duração média de uma hora, com a finalidade de informar e orientar sobre aspectos nutricionais, tais como: pirâmide alimentar, densidade energética dos alimentos, importância dos macro e micronutrientes e sua relação com a saúde, composiçẫo nutricional dos alimentos, controle das porçôes, estratégias para se alimentar fora de casa, comidas "diet" vs "light", preparação da comida e frequência das refeiçóes. Náo foi prescrita nenhuma dieta com consumo calórico fixo, os adolescentes foram apenas orientados a reduzir seu consumo calórico e realizar uma dieta balanceada.

\section{Intervenção dos profissionais da Educação Física}

Foram realizadas palestras semanais, conduzidas pelos profissionais e acadêmicos de Educação Física, a fim de fornecer informaçóes e esclarecimentos sobre a prática de atividade física e seus benefícios (com duração média de uma hora), estimulá-los a praticar atividade física fora do horário de intervençáo e atividade física orientada com frequência mínima de três vezes por semana (segundas, quartas e sextas-feiras) e duração média de uma hora por sessão. Foram priorizados os exercícios para grupos musculares localizados, por exemplo, abdominais, flexôes de braço, agachamentos e exercícios com "medicine ball", (60-66\% FCmax) ( $-20 \%$ do tempo de intervenção), caminhada/corrida (54-59/75-84\% FCmax), respectivamente $(-30 \%)$ e o basquetebol $(82-89 \%$ FCmax) $(-50 \%)$. Nas primeiras duas semanas de intervenção, as atividades de basquetebol envolveram exercícios técnicos para facilitar a continuidade das intervençôes desse esporte, que envolveu o jogo propriamente dito. A escolha do basquetebol justifica-se pelo fato deste ser um esporte coletivo, que favorece a interação social entre os adolescentes, é motivador pelo aspecto da realização das cestas, é dinâmico e mantêm a FC elevada (em torno de 82-89\% FCmax na amostra avaliada). Além disso, envolve a habilidade de membros superiores (diferente do futebol, por exemplo), e tem menos contato entre os jogadores (diferente do handebol, por exemplo), reduzindo assim as chances de lesōes ${ }^{21}$. Ademais, em estudos pilotos, observou-se que os adolescentes preferiam mais essa atividade comparada a outros esportes coletivos.

Os valores de \%FCmax citados anteriormente foram obtidos nas intervençōes da terceira e quarta semanas para caracterização das intensidades das intervençōes. Nestas semanas foram realizadas duas intervençóes focando cada grupo de exercícios físicos, caminhada/ corrida, exercícios resistidos e basquete, sendo que os períodos de recuperação não foram considerados. Foi utilizado um frequencímetro para monitoramento dessas sessóes (Polar FT1, Polar Electro, Kempele, Finlândia), sendo as intensidades determinadas a partir da FCmáx obtida no teste Léger de 20 metros para avaliação da aptidão cardiorrespiratóriaa ${ }^{22-23}$.

\section{Intervenção médica}

Foi conduzida por um pediatra, por meio da realização de consultas individuais com os pais e adolescentes a fim de buscar informações sobre os hábitos alimentares desde o nascimento, com especial interesse sobre os primeiros meses de vida e o tempo de amamentação. Foram coletadas também informaçóes sobre o início do excesso de massa corporal e doenças que já haviam sido diagnosticadas antes da participação do adolescente no programa e que poderiam interferir em seus resultados, além de orientá-los sobre a importância de determinados hábitos e seus principais efeitos para o estado geral de saúde desses adolescentes.

\section{Intervenção com os responsáveis}

Antes do início da intervenção, após o primeiro, o segundo e o terceiro mês da mesma, os pais foram convidados para palestras com os profissionais envolvidos no estudo, a fim de esclarecê-los acerca da importância da família no processo de mudança dos hábitos alimentares e de atividade física. Além disso, o momento foi aproveitado para informar individualmente aos pais os resultados parciais de seus filhos. 


\section{Avaliações e instrumentos da pesquisa}

Foi realizada uma reuniáo com os interessados em participar do projeto para explicar os objetivos e os tipos de intervençôes, bem como sobre a liberdade de deixar de participar a qualquer momento se desejarem. Com os que inicialmente concordaram em participar, foi firmado um termo de compromisso livre e esclarecido, no qual todas estas informaçôes estavam explicitadas, juntamente com o preenchimento de uma anamnese dos participantes.

As avaliaçôes foram feitas em dois momentos em ambos os grupos: a) antes do início da pesquisa (Pré-teste); b) após um período de 16 semanas do pré-teste.

\section{Avaliações antropométricas, composição corporal, maturação sexual e aptidão física relacionada à saúde}

$\mathrm{Na}$ semana que antecedeu o início do PMTO e na semana seguinte ao seu término, os adolescentes passaram por uma bateria de avaliaçóes, as quais incluíram a medida da massa corporal, altura, IMC, circunferência de cintura (CC) e circunferência de quadril (CQ). A massa corporal foi medida por meio de uma balança da marca Welmy com capacidade para medir até $300 \mathrm{~kg}$ e precisão de $0,05 \mathrm{~kg}$. A altura foi medida em um estadiômetro Standard Sanny com capacidade de medir até 2,30 metros e precisão de 0,1 $\mathrm{cm}$. O IMC foi calculado a partir da divisão da massa corporal dos adolescentes pela sua altura ao quadrado. As CC e CQ foram medidas com o auxílio de uma fita não extensiva de marca WISO com capacidade de medir até dois metros e precisão de $0,1 \mathrm{~cm}$.

Esses testes incluíram também a avaliação da composiçấo corporal na qual foi utilizado um aparelho de bioimpedância elétrica, multifrequencial, octapolar da marca Biospace, modelo InBody 520. Os adolescentes foram aconselhados a seguirem as recomendações descritas por HEYWARD ${ }^{24} \mathrm{e}$ só participaram aqueles que afirmaram que as seguiram: jejum de no mínimo duas horas inclusive de água, urinar cerca de 30 minutos antes da avaliação; abster-se do consumo de bebidas cafeinadas ao longo das últimas 48 horas; evitar esforços físicos vigorosos ao longo das últimas 24 horas; por fim, não utilizar diuréticos ao longo dos últimos sete dias. Foram incluídas na análise as medidas da massa gorda absoluta (MGA), massa magra (MM), massa gorda relativa (MGR).
A maturação sexual foi avaliada de acordo com os estágios de TANNER ${ }^{25}$. Adolescentes identificados no estágio 1 foram considerados como pré-púberes, nos estágios 2 e 3 como púberes e nos estágios 4 e 5 como pós-púberes.

Além disso, foram avaliados os seguintes parâmetros da AFRS: a flexibilidade, a força/resistência da musculatura abdominal e a aptidão cardiorrespiratória. Para a medição da flexibilidade foi aplicado o teste de Sentar-e-alcançar com banco de Wells, no qual os adolescentes deveriam sentar-se com as pernas estendidas e tentar alcançar a maior distância levando as duas mãos, uma sobre a outra, para frente. A força/ resistência da musculatura abdominal foi avaliada a partir do teste de flexão de tronco, em que os adolescentes deveriam realizar o maior número de repetiçóes do exercício abdominal durante um período de 60 segundos ${ }^{26}$. A aptidão cardiorrespiratória foi medida a partir do teste de Léger de $20 \mathrm{~m}$, teste este que é máximo iniciado a $8,5 \mathrm{~km} / \mathrm{h}$ e com incrementos progressivos de $0,5 \mathrm{~km} / \mathrm{h}$ a cada minuto até que o sujeito atingisse a exaustão. Os adolescentes foram orientados durante as corridas de $20 \mathrm{~m}$ por um sinal sonoro. Foi utilizada na análise a medida estimada do consumo máximo de oxigênio $\left(\mathrm{VO}_{2 \max }\right)$ relativo ${ }^{22-23}$. No encontro que antecedeu os testes físicos os adolescentes passaram por um processo de familiarizaçáo com os procedimentos adotados.

\section{Análise estatística}

Foi testada a normalidade dos dados, a partir do teste de Shapiro-Wilk. Os resultados foram tratados por meio da estatística descritiva (mediana e amplitude interquartílica - P75-25) e inferencial (comparação entre momentos e grupos). Foi utilizada a análise não paramétrica de Wilcoxon para a comparação entre os dois momentos de acordo com o grupo (controle ou intervenção e sexo masculino e sexo feminino). A associação entre grupo e sexo com estágio de maturação sexual foi feita por meio do teste de Qui-quadrado. A significância foi pré-estabelecida em $5 \%$. Foram calculados os tamanhos de efeito (TE) ([média do pós-teste - média do pré-teste]/desvio padrão do pré-teste) para determinar a magnitude dos efeitos da intervençáo. A magnitude do TE foi classificada de acordo com $\operatorname{CoHEN}^{27}$ como: $\leq 0,20$ (trivial), entre 0,21 e 0,50 (pequeno), entre 0,51 e 0,80 (moderado) e > 0,80 (grande). 


\section{Resultados}

A mediana da idade para o GI foi de $12,0(3,0)$ anos para os meninos e 13,0 $(2,0)$ anos para as meninas, enquanto para o GC foi de $13,0(2,0)$ anos para os meninos e $13,5(4,0)$ anos para as meninas, sem diferença significativa $(p>0,05)$. Em relação à maturaçáo sexual do sexo masculino, 1 (4,0\%), 17 $(68,0 \%)$ e $7(28,0 \%)$ dos adolescentes do GI foram considerados pré-púberes, púberes e pós-púberes, respectivamente, enquanto $1(3,5 \%), 13(44,8 \%)$ e $15(51,7 \%)$ classificados como pré-púberes, púberes e pós-púberes, respectivamente, no GC. No sexo feminino, $9(27,3 \%)$ e 24 (72,7\%) foram classificadas como púberes e pós-púberes, respectivamente, enquanto $9(34,6 \%)$ e $17(65,4 \%)$ consideradas púberes e pós-púberes. Cabe observar que os dados de maturaçáo sexual foram obtidos de uma amostra parcial dos adolescentes avaliados $(n=113 ; 69,3 \%)$.

Na TABELA 1 estáo os resultados dos 81 adolescentes do sexo masculino participantes do GI ( $\mathrm{n}=$ 45) e do GC ( $(n=36)$ antes e após o período de 16 semanas para as variáveis antropométricas, composição corporal e AFRS. Nota-se para o grupo GI melhora significativa nas variáveis IMC, CC, CQ, gordura relativa e absoluta e massa magra. Nos parâmetros de aptidão física, foram identificadas diferenças significativas nas três variáveis estudadas, sendo elas o $\mathrm{VO}_{2 \max }$, a flexibilidade e a força/resistência abdominal. Por outro lado no GC houve aumento da massa corporal, gordura relativa, massa magra, flexibilidade e no número de repetiçóes no teste abdominal.

Na TABELA 2 estáo os resultados das 82 adolescentes do sexo feminino participantes do GI (52) e do GC (30) antes e após o período de 16 semanas para as variáveis antropométricas, composição corporal e AFRS. Observa-se para o grupo GI melhora significativa nas variáveis IMC, CC, CQ, gordura relativa e absoluta e massa magra. Em relação aos parâmetros de aptidão física, foram identificadas diferenças significativas no $\mathrm{VO}_{2 \max }$, na flexibilidade e na força/resistência abdominal. Já no GC houve aumento da CQ e da flexibilidade.

Os dados apresentados demonstram que o PMTO promoveu melhoras importantes nas variáveis antropométricas, composição corporal e aptidão física tanto para os meninos quanto para as meninas, no entanto, é possível observar que as meninas apresentaram tamanho de efeito maior para as variáveis $\mathrm{CC}$, gordura absoluta, $\mathrm{VO}_{2 \max }$ e forçal resistência abdominal em comparação aos meninos. Assim, considerando o cálculo do tamanho de efeito, sugere-se resultados mais expressivos para as meninas em relação a essas variáveis.

TABELA 1 - Parâmetros antropométricos, aptidão física, composição corporal antes e após 16 semanas de intervenção para o sexo masculino.

\begin{tabular}{|c|c|c|c|c|c|c|}
\hline \multirow[b]{3}{*}{ Variável } & \multicolumn{6}{|c|}{ Masculino } \\
\hline & \multicolumn{3}{|c|}{ Grupo Intervençáo $(n=45)$} & \multicolumn{3}{|c|}{ Grupo Controle $(n=36)$} \\
\hline & Pré-teste & Pós-16 semanas & TE & Pré-teste & Pós-16 semanas & TE \\
\hline Massa corporal (kg) & $73,6(35,7)$ & $74,1(36,4)$ & $-0,00(\mathrm{~T})$ & $79,6(26,6)$ & $80,5(26,9)^{*}$ & $0,06(\mathrm{~T})$ \\
\hline Altura (m) & $1,56(0,17)$ & $1,59(0,17)^{*}$ & $0,00(\mathrm{~T})$ & $1,66(0,17)$ & $1,69(0,15)^{*}$ & $0,18(\mathrm{~T})$ \\
\hline $\operatorname{IMC}\left(\mathrm{kg} / \mathrm{m}^{2}\right)$ & $29,2(7,3)$ & $28,8(6,9)^{*}$ & $-0,13(\mathrm{~T})$ & $27,8(7,7)$ & $28,1(7,0)$ & $-0,05(\mathrm{~T})$ \\
\hline $\mathrm{CC}(\mathrm{cm})$ & $91,5(13,5)$ & $89,2(14,4)^{*}$ & $-0,17(\mathrm{~T})$ & $90,8(16,4)$ & $89,3(15,1)$ & $-0,04(\mathrm{~T})$ \\
\hline $\mathrm{CQ}(\mathrm{cm})$ & $105,5(19,8)$ & $103,0(21,0)^{*}$ & $-0,12(\mathrm{~T})$ & $104,0(16,1)$ & $104,4(17,6)$ & $-0,00(\mathrm{~T})$ \\
\hline Gordura (\%) & $43,5(12,1)$ & $41,9(12,7)^{*}$ & $-0,38(\mathrm{P})$ & $40,6(8,0)$ & $41,0(7,2)^{*}$ & $-0,15(\mathrm{~T})$ \\
\hline Gordura (kg) & $32,3(17,3)$ & $30,3(14,3)^{*}$ & $-0,15(\mathrm{~T})$ & $29,2(14,6)$ & $27,6(15,5)$ & $-0,06(\mathrm{~T})$ \\
\hline Massa magra (kg) & $37,9(15,5)$ & $41,1(18,8)^{*}$ & $0,17(\mathrm{~T})$ & $45,8(10,8)$ & $46,8(10,9)^{*}$ & $0,16(\mathrm{~T})$ \\
\hline $\mathrm{VO}_{2 \max }(\mathrm{mL} / \mathrm{kg} / \mathrm{min})$ & $23,8(5,10)$ & $26,0(9,7)^{*}$ & $0,39(\mathrm{P})$ & $24,9(7,5)$ & $27,0(6,8)$ & $0,07(\mathrm{~T})$ \\
\hline Flexibilidade $(\mathrm{cm})$ & $19,0(13,0)$ & $22,5(16,0)^{*}$ & $0,36(\mathrm{P})$ & $19,0(14,0)$ & $20,5(16,0)^{*}$ & $0,20(\mathrm{~T})$ \\
\hline Abdominal (repetições) & $19,0(13,0)$ & $28,0(13,0)^{*}$ & $0,80(\mathrm{M})$ & $22,0(12,0)$ & $25,0(14,0)^{*}$ & $0,33(\mathrm{P})$ \\
\hline
\end{tabular}

Dados apresentados em mediana (amplitude interquartílica). IMC: índice de massa corporal; CC: circunferência de cintura;

$C Q$ : circunferência de quadril;

$\mathrm{VO}_{2 \text { max }}$ : consumo máximo de oxigênio;

TE: tamanho de efeito; T: TE trivial;

$P$ : TE pequeno;

M: TE moderado;

G: TE grande.

*Diferença significativa considerando o Pré-teste dentro do mesmo grupo. 
TABELA 2 - Parâmetros antropométricos, aptidão física, composição corporal antes e após 16 semanas de intervenção para o sexo feminino.

Dados apresentados em mediana (amplitude interquartílica). IMC: índice de massa corporal;

CC: circunferência de cintura;

$\mathrm{CQ}$ : circunferência de quadril;

$\mathrm{VO}_{2 \text { áx }}$ : consumo máximo de oxigênio;

TE: tamanho de efeito;

T: TE trivial;

$P$ : TE pequeno;

M: TE moderado

G: TE grande.

*Diferença significativa

considerando o Pré-teste

dentro do mesmo grupo.

\begin{tabular}{|c|c|c|c|c|c|c|}
\hline \multirow[b]{3}{*}{ Variável } & \multicolumn{6}{|c|}{ Feminino } \\
\hline & \multicolumn{3}{|c|}{ Grupo Intervençáo $(\mathrm{n}=52)$} & \multicolumn{3}{|c|}{ Grupo Controle $(\mathrm{n}=30)$} \\
\hline & Pré-teste & Pós-16 semanas & TE & Pré-teste & Pós-16 semanas & TE \\
\hline Massa corporal (kg) & $79,0(25,8)$ & $77,5(22,2)$ & $-0,02(\mathrm{~T})$ & $73,8(15,6)$ & $72,4(17,2)$ & $0,04(\mathrm{~T})$ \\
\hline Altura (m) & $1,60(0,08)$ & $1,61(0,08)^{*}$ & $0,01(\mathrm{~T})$ & $1,57(0,13)$ & $1,59(0,13)^{*}$ & $0,13(\mathrm{~T})$ \\
\hline $\operatorname{IMC~}\left(\mathrm{kg} / \mathrm{m}^{2}\right)$ & $31,0(7,90)$ & $30,2(7,8)^{*}$ & $-0,15(\mathrm{~T})$ & $28,7(5,9)$ & $28,8(7,0)$ & $-0,02(\mathrm{~T})$ \\
\hline $\mathrm{CC}(\mathrm{cm})$ & $87,3(13,8)$ & $86,0(13,5)^{*}$ & $-0,26(\mathrm{P})$ & $85,3(11,7)$ & $85,2(10,4)$ & $-0,05(\mathrm{~T})$ \\
\hline CQ $(\mathrm{cm})$ & $110,0(13,5)$ & $109,9(13,1)^{*}$ & $0,02(\mathrm{~T})$ & $107,5(15,9)$ & $109,5(15,6)^{*}$ & $0,27(\mathrm{P})$ \\
\hline Gordura (\%) & $45,7(8,7)$ & $44,4(10,7)^{*}$ & $-0,26(\mathrm{P})$ & $45,4(5,3)$ & $46,0(5,6)$ & $0,11(\mathrm{~T})$ \\
\hline Gordura (kg) & $36,0(16,5)$ & $34,2(15,5)^{*}$ & $-0,26(\mathrm{P})$ & $31,2(10,1)$ & $31,3(14,3)$ & $0,02(\mathrm{~T})$ \\
\hline Massa magra $(\mathrm{kg})$ & $39,1(10,6)$ & $41,3(8,9)^{*}$ & $0,19(\mathrm{~T})$ & $37,5(9,0)$ & $37,6(7,4)$ & $0,05(\mathrm{~T})$ \\
\hline $\mathrm{VO}_{2 \max }(\mathrm{mL} / \mathrm{kg} / \mathrm{min})$ & $23,0(2,9)$ & $25,7(4,5)^{*}$ & $0,83(\mathrm{G})$ & $24,5(6,4)$ & $23,8(5,9)$ & $-0,14(\mathrm{~T})$ \\
\hline Flexibilidade $(\mathrm{cm})$ & $27,0(10,0)$ & $30,8(9,0)^{*}$ & $0,37(\mathrm{P})$ & $26,0(10,0)$ & $30,0(11,0)^{*}$ & $0,35(\mathrm{P})$ \\
\hline Abdominal (repetições) & $18,0(12,0)$ & $27,0(15,0)^{*}$ & $1,01(\mathrm{G})$ & $20,0(12,0)$ & $20,0(14,0)$ & $0,09(\mathrm{~T})$ \\
\hline
\end{tabular}

\section{Discussão}

O objetivo do presente estudo foi verificar as diferenças entre as respostas de meninos e meninas a um programa multiprofissional de tratamento da obesidade (PMTO) sobre a AFRS. Os principais achados deste estudo são que um PMTO com duração de 16 semanas promoveu efeitos positivos nas variáveis antropométricas, composição corporal e AFRS tanto para meninos quanto para meninas, sendo encontrados tamanhos de efeito superiores para as meninas nas variáveis $\mathrm{CC}$, gordura absoluta, $\mathrm{VO}_{2 \max }$ e força/resistência abdominal. Em contrapartida, no grupo GC os meninos apresentaram aumento significativo da massa corporal, gordura relativa, massa magra, flexibilidade e no número de repetiçóes no teste de abdominal enquanto as meninas aumento da CQ e flexibilidade.

Intervenções multiprofissionais de curto/médio prazo para adolescentes com excesso de gordura corporal têm demonstrado melhora na antropometria, composição corporal e AFRS ${ }^{9-10,12-13,19,28-30}$. Quanto às diferenças entre os sexos, BIANCHINI et al. ${ }^{29}$ encontraram alteraçôes significativas nas variáveis IMC, CC, CQ, gordura (kg) e massa magra $(\mathrm{kg})$ em ambos os sexos após 16 semanas de intervenção multiprofissional, no entanto, os tamanhos de efeito para o sexo feminino foram superiores aos observados para o sexo masculino (pequeno vs trivial), com exceção da massa magra, na qual se observou o contrário. Coppen et al. ${ }^{30}$ observaram redução significativa da CC, independente do sexo, após 10 semanas de um programa para controle da obesidade realizado com 135 crianças e adolescentes obesos. Por outro lado, no estudo de CARANTi et al. ${ }^{19}$, apenas os meninos apresentaram mudanças significativas no IMC e gordura corporal (\%).

Para os conflitantes resultados desses estudos, pode-se levantar a hipótese de que diferenças na faixa etária de maturação sexual poderiam contribuir, tendo em vista que as demais características das amostras estudadas ou da intervenção realizada eram semelhantes. BIANCHINI et al. ${ }^{29}$ avaliaram adolescentes com idade entre 10 e 18 anos, sendo que $34,8 \%$ das meninas e $50 \%$ dos meninos do grupo intervenção eram púberes. Coppen et al. ${ }^{30}$ analisaram uma faixa etária ainda mais ampla, com idade variando entre seis e 19 anos, em que possivelmente todos os estágios de maturação sexual estavam presentes. Diferentemente desses estudos, CARANTI et al. ${ }^{19}$ incluíram em sua amostra apenas adolescentes no estágio pós-púbere. Diante dessas diferenças, em somatório com os resultados obtidos no presente estudo para as variáveis antropométricas e da composição corporal, parece que diferentes faixas etárias e estágio de maturação sexual podem influenciar nas respostas de um programa multiprofissional de intervenção. No entanto, essa hipótese precisa ser mais bem estudada. 
Apesar das diferenças entre sexos nas variáveis antropométricas e composição corporal, mencionadas acima, para as demais variáveis de AFRS, a partir do que se foi levantado na literatura, este é o primeiro estudo que investigou se as respostas entre meninos e meninas são diferentes após um período de intervenção multiprofissional para o tratamento da obesidade em adolescentes. Segundo Malina e Bouchard ${ }^{15}$, no período da puberdade as meninas tendem a aumentar a gordura enquanto os meninos a massa muscular. Essas diferenças geralmente refletem em melhores níveis de aptidão física para os meninos, com exceção da flexibilidade. No entanto, no presente estudo, tanto as meninas quanto os meninos apresentaram melhora significativa nas variáveis gordura relativa e absoluta, massa magra, $\mathrm{VO}_{2 \max }$, flexibilidade e força/ resistência abdominal após o período de intervenção multiprofissional. Porém, considerando os tamanhos de efeitos, observa-se que as meninas obtiveram resultados mais expressivos nas variáveis gordura absoluta (TE pequeno), $\mathrm{VO}_{2 \max }$, (TE grande) e força/ resistência abdominal (TE grande) em comparação aos meninos que apresentaram tamanhos de efeito trivial, pequeno e moderado, respectivamente.

Uma possível explicação para as diferenças encontradas no presente estudo quanto à magnitude do efeito proporcionado pela intervenção poderia estar relacionada a estas diferenças entre meninos e meninas quanto ao nível de aptidão física, no qual o sexo que apresenta menores níveis tenderia a apresentar melhoras mais expressivas. No entanto, os resultados apontam não haver diferença entre meninos e meninas para as variáveis de aptidão física, no pré-teste, com exceção da flexibilidade. A obesidade pode ser considerada um fator que contribui para a redução dos níveis de aptidão física, independente do sexo ${ }^{31}$. Assim, considerando que não houve diferenças entre os sexos no início da intervenção para as variáveis estudadas, outros fatores poderiam explicar as diferenças encontradas.

Estudos demonstram que os homens, especialmente na adolescência, não sofrem tanta pressão social, apresentando uma melhor aceitação de seu o corpo ${ }^{32}$. Por outro lado as meninas costumam apresentar maior insatisfação corporal, maior preocupação com a imagem corporal e um maior desejo de diminuir a massa corporal ${ }^{33-34}$, o que pode contribuir para um maior comprometimento e dedicação das meninas nas intervençóes. Outro fator que poderia influenciar nas diferenças entre os sexos é a maturação sexual dos adolescentes, que pode promover mudanças fisiológicas ${ }^{15}$, especialmente as relacionadas à distribuição de gordura corporal ${ }^{19}$. A proporção de meninas pós-púberes no GI foi superior à proporção de meninos no mesmo estágio para o mesmo grupo. Estudos feitos apenas com meninas e meninos pós-púberes revelaram perda da gordura corporal mais acentuada ${ }^{19}$ comparado com estudos feitos com adolescentes em diferentes estágios de desenvolvimento puberal ${ }^{29}$. É importante ressaltar que, apesar de serem estudos conduzidos por equipes multiprofissionais, as intervençôes dos profissionais de educação física não foram exatamente as mesmas.

As mudanças observadas no GC, em especial o aumento da CQ nas meninas e o aumento da gordura corporal (\%) nos meninos reforça a importância da participação em um PMTO, visto que a manutenção do estilo de vida desses adolescentes pode levar ao agravamento do quadro de obesidade, que se náo controlado, aumenta as chances do sujeito ser obeso na fase adulta ${ }^{35}$ e consequentemente, desenvolver doenças cardiovasculares $^{36}$. As melhoras obtidas na flexibilidade em ambos os sexos e na força/resistência abdominal no sexo masculino podem estar relacionadas ao desenvolvimento físico dessa faixa etária ${ }^{15}$, basta notar o aumento na estatura e massa muscular (esta para os meninos).

Apesar das contribuiçóes do presente estudo em relação à investigação das respostas de meninos e meninas a um PMTO em relação à AFRS, este apresenta como limitação a não randomização da amostra. No entanto, a forma de seleçáo da amostra por conveniência, tem como ponto positivo o oferecimento do tratamento para todos os adolescentes que procuraram atendimento e apresentavam disponibilidade para a participação integral das intervençôes. Somado a isso, não foi feita a medida da prática de atividade física fora do ambiente do programa multiprofissional, portanto, não se sabe se as mudanças na AFRS se deram mais pela prática regular oferecida durante o programa ou se a intervenção da equipe multiprofissional, mesmo que sem a prática regular de exercícios, poderia promover mudanças nessas variáveis. Sugere-se para futuros estudos dentro dessa perspectiva, a identificação dos principais fatores que explicariam os melhores resultados obtidos pelas meninas para algumas variáveis da AFRS.

Em resumo, com os resultados deste estudo, conclui-se que 16 semanas de intervenção multiprofissional foram suficientes para promover melhora em parâmetros antropométricos, de composiçáo corporal eAFRS tanto de meninos quanto de meninas com sobrepeso ou obesidade, destacando-se o sexo feminino quanto à magnitude do efeito na gordura absoluta, $\mathrm{VO}_{2 \max }$, e na força/resistência abdominal. 


\begin{abstract}
Multiprofessional intervention improves health-related physical fitness in adolescents with greater effect on girls compared to boys

This study aimed to verify the differences between both boys and girls responses to a multidisciplinary program of obesity treatment on health-related physical fitness. One hundred and sixty three adolescents with excess body weight and aged 10 to 18 years took part in the present study. It was assessed anthropometric parameters, body composition, flexibility, abdominal strength/resistance and cardiorespiratory fitness before and after 16 weeks of a multiprofessional intervention. The multiprofessional intervention was developed by physical educators, nutritionist, psychologist, pediatrician and was focused on motivate eating and exercise changes which could promote positive changes on the parameters analyzed. The intervention was conducted three times per week in which theoretical intervention lasted one hour with physical educators, nutritionist and psychologist and another hour designated to practical interventions of the physical educators in which predominated resistance and aerobic exercise as well as basketball practice. After the intervention, both girls and boys presented significant improvements in BMI, waist and hip circumference, relative and absolute body fat, lean mass, flexibility, strength and resistance in abdominal and cardiorespiratory fitness; however the results were more expressive for girls on waist circumference, body fat $(\mathrm{kg}), \mathrm{VO}_{2 \max }$ and abdominal strength/resistance.
\end{abstract}

KeY Words: Adolescent; Obesity; Physical fitness; Intervention study.

\title{
Referências
}

1. Christodoulos AD, Douda HT, Tokmakidis SP. Cardiorespiratory fitness, metabolic risk, and inflammation in children. Int J Pediatr. 2012;2012:2705-15.

2. Maggio AB, Hofer MF, Martin XE, Marchand LM, Beghetti M, Farpour-Lambert NJ. Reduced physical activity level and cardiorespiratory fitness in children with chronic diseases. Eur J Pediatr. 2010;169:1187-93.

3. Aires L, Silva P, Silva G, Santos MP, Ribeiro JC, Mota J. Intensity of physical activity, cardiorrespiratory fitness, and body mass index in youth. J Phys Act Health. 2010;7:54-9.

4. Bailey DP, Fairclough SJ, Savory LA, et al. Accelerometry-assessed sedentary behaviour and physical activity levels during the segmented school day in 10-14-year-old children: the HAPPY study. Eur J Pediatr. 2012;171:1805-13.

5. Bouglé D, Zunquin G, Sesbouë B, Sabatier JP. Relationships of cardiorespiratory fitness with metabolic risk factors, inflammation, and liver transaminases in overweight youths. Int J Pediatr. 2010;2010:580897.

6. Daniels SR, Greer FR. Lipid screening and cardiovascular health in childhood. Pediatrics. 2008;122:198-208.

7. Andersen LB, Harro M, Sardinha LB, et al. Physical activity and clustered cardiovascular risk in children: a cross-sectional study (The European Youth Heart Study). Lancet. 2006;368:299-304.

8. Nassis GP, Papantakou K, Skenderi K, et al. Aerobic exercise training improves insulin sensitivity without changes in body weight, body fat, adiponectin, and inflammatory markers in overweight and obese girls. Metabolism. 2005;54:1472-9.

9. Silva DF, Lima LL, Delfino RO, Bianchini JAA, Hintzie LJ, Nardo Junior N. Efeitos de um programa multiprofissional de tratamento da obesidade e de sua cessação sobre a aptidão física relacionada à saúde de adolescentes. Rev Educ Fis/ UEM. 2012;23:399-410.

10. Poeta LS, Duarte MFS, Giuliano ICB, Farias Junior JC. Intervenção interdisciplinar na composição corporal e em testes de aptidão física de crianças obesas. Rev Bras Cineantropom Desempenho Hum. 2012;14:134-43.

11. Bianchini JA, Silva DF, Nardo CC, Carolino ID, Hernandes F, Nardo Junior N. Multidisciplinary therapy reduces risk factors for metabolic syndrome in obese adolescents. Eur J Pediatr. 2013;172:215-21.

12. Leite N, Milano GE, Cielask F, Lopes WA, Rodacki A, Radominski RB. Effects of physical exercise and nutritional guidance on metabolic syndrome in obese adolescents. Rev Bras Fisioter. 2009;13:73-81.

13. Carrel AL, Clark RR, Peterson SE, Nemeth BA, Sullivan J, Allen DB. Improvement of fitness, body composition, and insulin sensitivity in overweight children in a school-based exercise program: a randomized, controlled study. Arch Pediatr Adolesc Med. 2005;159:963-8.

1058 • Rev Bras Educ Fís Esporte, (São Paulo) 2016 Out-Dez; 30(4):1051-59 
14. Sabia RV, Santos JE, Ribeiro RPP. Efeito da atividade física associada à orientação alimentar em adolescentes obesos: comparação entre o exercício aeróbio e anaeróbio. Rev Bras Med Esporte. 2004;10:349-55.

15. Malina RM, Bouchard C. Atividade física do atleta jovem: do crescimento à maturação. São Paulo: Roca; 2002.

16. Daniels SR, Arnett DK, Eckel RH, et al. Overweight in children and adolescents: pathophysiology, consequences, prevention, and treatment. Circulation. 2005;111:1999-2012.

17. Cole TJ, Lobstein T. Extended international (IOTF) body mass index cut-offs for thinness, overweight and obesity. Pediatr Obes. 2012:7:284-94.

18. Leite N, Lazarotto L, Cavazza JF, et al. Efeitos de exercícios aquáticos e orientação nutricional sobre a composição corporal de adolescentes obesos. Rev Bras Cineantropom Desempenho Hum. 2010;12:232-8.

19. Caranti DA, de Mello MT, Prado WL, et al. Short- and long-term beneficial effects of a multidisciplinary therapy for the control of metabolic syndrome in obese adolescents. Metabolism. 2007;56:1293-300.

20. Cooper Z, Fairburn CG, Hawker DM. Cognitive-behavioral treatment of obesity. New York: Guildford Press; 2003.

21. Villas Boas MS. Basquetebol brincando e aprendendo: da iniciação ao aperfeiçoamento. Maringá: Eduem; 2008.

22. Leger LA, Lambert J. A maximal multistage 20m shuttle run test to predict $\mathrm{VO}_{2 \max }$. Eur J Appl Physiol. 1982;49:1-5.

23. Leger LA, Mercier D, Gadoury C, Lambert J. The multistage 20 metre shuttle run test for aerobic fitness. J Sports Sci. 1988;6:93-101.

24. Heyward VH. ASEP methods recommendation: body composition assessment. J Exerc Physiol. 2001;4:1-12.

25. Tanner JM. Normal growth and techniques of growth assessment. Clin Endocrinol Metab. 1986;15:411-51.

26. Gaya A, Silva G, editores. Projeto esporte Brasil: manual de aplicação de medidas e testes, normas e critérios de avaliação. Porto Alegre: PROESP-BR; 2007.

27. Cohen J. Statistical power analysis for the behavioral sciences. Hillsdale: Lawrence Erlbaum; 1988.

28. Hintze LJ, Cattai GBP, Nardo Junior N. Multidisciplinary program for obesity treatment: summary of results with adolescents. Acta Sci Health Sci. 2012;34:137-44.

29. Bianchini JAA, da Silva DF, Lopera CA, Matsuo AR, Antonini VD, Nardo Junior N. Efectos de un programa multiprofesional de tratamiento de la obesidad sobre los factores de riesgo para síndrome metabólico en niños prepúberes, púberes y adolescentes: diferencias entre géneros. Rev Andal Med Deporte. 2013;6:139-45.

30. Coppen AM, Risser JA, Vash PD. Metabolic syndrome resolution in children and adolescents after 10 weeks of weight loss. J Cardiometab Syndr. 2008;3:205-10.

31. Malina RM, Bouchard C, Bor-Or O. Growth, maturation, and physical activity. Champaign: Human Kinetics; 2004.

32. Graham MA, Eich C, Kephart B, Peterson D. Relationship among body image, sex, and popularity of high school students. Percept Mot Skills. 2000;90:1187-93.

33. Duca GF, Garcia LMT, Sousa TF, Oliveira ESA, Nahas MV. Insatisfação com o peso corporal e fatores associados em adolescentes. Rev Paul Pediatr. 2010;28:340-6.

34. Al Sabbah H, Vereecken C, Abdeen Z, Coats E, Maes L. Associations of overweight and of weight dissatisfaction among Palestinian adolescents: findings from the national study of Palestinian schoolchildren (HBSC-WBG2004). J Hum Nutr Diet. 2009;22:40-9.

35. Wijga AH, Scholtens S, Bemelmans WJ, et al. Comorbidities of obesity in school children: a cross-sectional study in the PIAMA birth cohort. BMC Public Health. 2010;10:184.

36. Cheung YB, Machin D, Karlberg J, Khoo KS. A longitudinal study of paediatric BMI values predicted health in middle age. J Clin Epidemiol. 2004;57:1316-22.

\section{Agradecimentos}

Os autores agradecem às fontes de financiamento: Fundação Araucária e CAPES.

ENDEREÇO

Josiane Aparecida Alves Bianchini

Departamento de Educação Física

Universidade Estadual de Maringá

Av. Colombo, 5700 - Bloco M o6 - sala 6

87020-900 - Maringá - PR - BRASIL e-mail: josi_edf@hotmail.com
Recebido para publicação: 13/12/2013

1a. Revisão: 25/02/2014

2a. Revisão: 01/07/2015

Aceito: $17 / 07 / 2015$ 